\title{
Giovani nefrologi che fanno ricerca: quando scienza e passione si incontrano
}

\author{
Davide Bolignano
}

CNR-Institute of Clinical Physiology, Reggio Calabria

YOUNG NEPHROLOGISTS DOING RESEARCH: WHEN SCIENCE MEETS PASSION

Abstract. Scientific research represents the future of nephrology, and tomorrow's research belongs to the nephrologists of tomorrow. Young nephrologists might have several opportunities for conducting research. However, good ideas and accumulating experience are not the only keys for success: science always needs to meet passion and curiosity. We herein present the story of a young nephrologist and his research experiences, from the first steps to the first achievements, as a way of proving to other young colleagues that scientific enthusiasm and devotion to science sometimes make dreams become true.

Key words: Scientific research, Nephrology, Young nephrologists

Conflict of interest: None.

Financial support: None.

Accettato: 12 Dicembre 2014

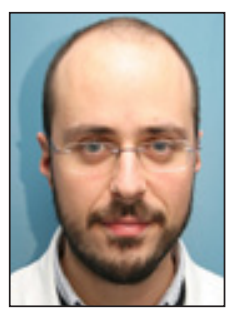

Davide Bolignano

\section{I primi passi}

Ho sempre amato la ricerca, fin da quando ero un semplice studente di medicina. Credo di aver scelto la strada della nefrologia in parte anche per questo. Quale altra specialità spazia infatti, al pari della nefrologia, dall'immunologia alla medicina cardiovascolare, dall'anatomia patologica alla medicina interna, dal banco di laboratorio al letto del paziente.

La passione per la ricerca è definitivamente scoppiata durante gli anni di specializzazione. Ricordo ancora pomeriggi interi (e talvolta anche sere e notti) passate a raccogliere dati, "tentare" elaborazioni statistiche, intervistare pazienti o collezionare campioni di urine o sangue da centrifugare. Come specializzandi, anche nella ricerca facevamo un po' di tutto ma, per chi come me era appassionato, il sacrificio di spendere in questo modo il "tempo libero" veniva sempre puntualmente ripagato. Ricordo bene il primo abstract accettato come poster e la paura (ampiamente immotivata) di doverlo presentare in occasione del mio primo congresso SIN (Roma 2006). Da lì alla prima comunicazione orale (ancora più paura, ancora più immotivata!) il passo è stato breve e così, poster dopo poster, comunicazione dopo comunicazione le soddisfazioni ottenute hanno sempre più alimentato il fuoco dell'entusiasmo.

Ma l'entusiasmo non è tutto, per fare ricerca ci vuole anche (un po') di iniziativa, un link di conoscenze e, soprattutto direi, la giusta formazione. Nonostante non disdegnassi qualche interessante e originale affaccio nella nefrologia di base (con qualche lavoretto su cellule ed animali davvero non male!), la mia vocazione si è fin da subito orientata sull'epidemiologia e la nefrologia clinica. Grazie alla Società Italiana di Nefrologia (SIN) ho ottenuto una mini borsa di studio che mi ha permesso, nel 2008, di approfondire le mie conoscenze epidemiologiche in un corso organizzato dall'ERA-EDTA a Bristol. La partecipazione attiva alla vita della Società, in particolare a congressi e gruppi di studio della SIN, mi ha poi progressivamente permesso di creare un'ottima rete di rapporti personali nonché un diretto coinvolgimento in studi clinici multicentrici promossi dalla florida realtà italiana. All'interno della SIN, oggi collaboro prevalentemente con il gruppo di studio sull'esercizio fisico nel paziente nefropatico: un topic di cui, ne sono sicuro, si sentirà parlare spesso in futuro e sul quale noi italiani siamo in grado di dire la nostra, più di chiunque altro.

Il tempo passa e ho iniziato ad aggiungere sempre più mattoncini alla mia carriera da ricercatore: congressi, corsi di epidemiologia, abstracts, pubblicazioni. Iniziano ad arrivare persino le prime letture su invito, prevalentemente nell'ambito dei biomarcatori nelle nefropatie acute e croniche. Realizzo in maniera sempre più convinta che la ricerca sta diventando davvero la mia strada (sempre però strizzando un occhio alla clinica e ai numerosi, affezionati pazienti che non avrebbero mai accettato l'idea di non essere più visitati dal loro nefrologo preferito).

\section{I primi traguardi}

Terminata la specializzazione ottengo un assegno di ricerca presso la sede del CNR della mia città, Reggio Calabria, che, come noto a molti, opera proprio nell'ambito della biomedicina, epidemiologia e statistica applicata alla nefrologia. Ricerca e clinica allo stesso tempo e, soprattutto, a casa. Un sogno per molti, ma un sogno da tenere stretto. E per ambire al passag- 
gio a ricercatore confermato (vincendo un concorso) gli abstracts e le pubblicazioni non bastano. Ci vuole il salto di qualità, mi dicono. E il salto di qualità arriva grazie a una call dell'ERAEDTA alla quale partecipo un po' per scherzo. Il progetto è ambizioso: ERA-EDTA vuole "rifondare" con uno spirito nuovo il gruppo di lavoro ERBP (European Renal Best Practice) che si occupa della redazione delle linee guida europee di nefrologia. A tale scopo si cercano nuovi, giovani fellows da formare appositamente nella metodologia della ricerca e della valutazione della letteratura scientifica, delle revisioni sistematiche e delle metaanalisi, ovvero il fondamento delle moderne linee guida. Ricordo benissimo il momento in cui mi viene comunicato di essere stato scelto (insieme a due soli altri colleghi, una belga e un rumeno) ad occupare questo ruolo. Neanche il tempo di rendermene conto e devo fare le valigie per "molto molto lontano". Sydney, destinazione Cochrane Renal Group: praticamente il massimo per un giovane nefrologo che sogna di fare ricerca a un certo livello. A Sydney imparo tanto e conosco un sacco di giovani che, come me, amano la ricerca nefrologica. Creare un link di conoscenze è importante, come dicevo, ed io continuo tutt'oggi a collaborare e a lavorare con i miei colleghi e amici di "down-under" (e così che, da molti, viene definita l'Australia), nonostante siano passati ormai un paio di anni da quando ho ultimato il mio training nella terra dei canguri. $\mathrm{Al}$ mio ritorno, una bella novità e una bella svolta: vinco il tanto agognato concorso da ricercatore (grazie anche al lavoro e all'esperienza accumulata nell'altro emisfero) ma adesso mi tocca lavorare davvero duro, in particolare per ERBP. Sono coinvolto nella stesura delle prossime linee guida sulla gestione del paziente diabetico nefropatico e su una serie di altri progetti satellite che includono anche una parte "didattica" e di disseminazione scientifica. Ma la posta è alta ed ERBP ci tiene ad investire nella formazione e nella crescita scientifica dei suoi fellows (che nel frattempo sono aumentati di 2 unità!). Decido perciò di cogliere una nuova occasione al volo e grazie a un cospicuo investimento finanziario di ERBP, che paga i suoi fellows non in moneta sonante ma in "accrescimento" professionale, decido di partecipare al prestigioso Global Clinical Scholars Research Training Program in Clinical Trials tenuto dall'Università di Harvard, tra Londra e Boston. Il programma è davvero tosto. Abbiamo un anno per padroneggiare la scienza (imperfetta) dei trials clinici: dal design alla statistica, dall'etica all'economia, per culminare nella tesi finale in cui devo progettare un vero e proprio (e soprattutto credibile) trial clinico da sottoporre all'attenzione di una temibile commissione composta da editors del prestigioso New England Journal of Medicine. Tra mille sacrifici (e tempo da trovare) riesco a concludere con successo il master ottenendo addirittura un "commitment" per l'originalità della mia tesi, che non a caso è su un argomento di nefrologia.

La sete per la ricerca non si placa e la voglia di conoscere altri colleghi, specie giovani, con cui condividere sogni, esperienze e, perché no, progetti mi accompagna quotidianamente. Partecipo a un'altra call dell'ERA-EDTA che ha in programma la costituzione di una piattaforma di giovani nefrologi europei (la Young Nephrologists Platform, YNP) e, tramite voto elettronico di tutti i membri ERA-EDTA under 40, mi ritrovo addirittura eletto nel consiglio direttivo (Board) di YNP insieme ad altri 5 giovani nefrologi provenienti da altrettanti Paesi europei. Anche questo compito è arduo: creare un sistema che metta in rete i migliori giovani nefrologi europei e ne promuova l'interazione, la crescita, la formazione e la realizzazione di progetti. Il futuro dirà se ci saremo, almeno in parte, riusciti. Nel frattempo, meglio tenere sempre un piede per terra. Decido quindi di prendere parte anche a un paio di working groups dell'ERA-EDTA, tra cui EURECAM ed EURORECKD (rispettivamente medicina cardiovascolare nella nefrologia e riabilitazione nella $\mathrm{CKD}$, cioè l'equivalente del gruppo di studio italiano sull'esercizio fisico nel paziente nefropatico), per tenere sempre aperta la possibilità di essere coinvolto in iniziative scientifiche e studi clinici multicentrici, la mia grande passione.

\section{Conclusioni}

L'importante, mi dico sempre, è non fermarsi mai e non pensare mai di essere arrivati, soprattutto professionalmente. È proprio in quel momento che al fuoco della ricerca si sostituisce l'apatia dell'appiattimento. Così come per ogni giovane nefrologo è impossibile conoscere cosa mi riserverà il futuro. Ma il futuro non si predice, si costruisce. E per un giovane nefrologo che ha voglia di fare buona ricerca le occasioni, particolarmente nell'ambito di SIN ed ERA-EDTA, sono davvero tante. A volte persino più vicine di quanto si possa immaginare. Basta solo saperle cogliere, credere nei propri mezzi e non mollare mai.

\section{Riassunto}

La ricerca scientifica rappresenta il futuro della nefrologia e la ricerca del domani appartiene ai nefrologi del domani. I giovani nefrologi possono avere diverse opportunità per coltivare la ricerca. Le buone idee e l'esperienza accumulata, tuttavia, non sono le sole chiavi per il successo. La scienza deve sempre incontrare la passione e la curiosità. Presentiamo qui la storia di un giovane nefrologo con le sue esperienze personali di ricerca, dai primi passi ai primi traguardi, come modo per dimostrare agli altri giovani colleghi che l'entusiasmo scientifico e la devozione alla scienza spesso rendono i sogni realizzabili.

Parole chiave: Ricerca scientifica, Nefrologia, Giovani nefrologi

Dichiarazione di conflitto di interesse: L'Autore dichiara di non avere conflitto di interessi.

Contributi economici agli Autori: L'Autore dichiara di non avere ricevuto sponsorizzazioni economiche per la preparazione dell'articolo.

Indirizzo dell'Autore:

Dr. Davide Bolignano

CNR-Institute of Clinical Physiology

c/o EUROLINE

Via Vallone Petrara 55

89124 Reggio Calabria

davide.bolignano@gmail.com 